\title{
Proton structure and QCD dynamics at low x
}

\author{
N. Raičević on behalf of the H1 and ZEUS Collaborations ${ }^{\text {a }}$ \\ ${ }^{a}$ University of Montenegro, Faculty of Science, Cetinjski put BB, 81000 Podgorica, Montenegro
}

A brief overview is given on most recent, preliminary results obtained by the HERA experiments H1 and ZEUS on the measurement of the neutral current $e^{ \pm} p$ scattering cross section. The paper focuses on the first combinations and QCD analyses of joint H1 and ZEUS data, on an H1 measurement in the transition region from hadronic to partonic behavior and presents the first results obtained on the measurement of the longitudinal structure function $F_{L}$ at low Bjorken $x$ using recent data collected at reduced proton beam energies.

\section{INTRODUCTION}

Results from the HERA experiments, H1 and ZEUS, on $e^{ \pm} p$ deep inelastic scattering (DIS) provide important knowledge of the proton structure and QCD. The data were collected in the years 1994-2000 (HERA I) and 2003-2007 (HERA II) in the center of mass energy of $\sqrt{s}=300 \mathrm{GeV}$ $\left(E_{p}=820 \mathrm{GeV}, E_{e^{ \pm}}=27.5 \mathrm{GeV}\right)$ in 1994-1997 and $319 \mathrm{GeV}\left(E_{p}=920 \mathrm{GeV}, E_{e^{ \pm}}=27.5 \mathrm{GeV}\right)$ in the years 1998-2007. From April to June 2007 at HERA, two reduced proton beam energies were used, the smallest energy of $460 \mathrm{GeV}$ and an intermediate energy of $575 \mathrm{GeV}$ while the positron beam energy was kept at $27.5 \mathrm{GeV}$.

The inclusive DIS $e^{ \pm} p$ double-differential cross section at low values of $Q^{2}$, in the one-photon exchange approximation, can be expressed as

$\frac{d^{2} \sigma}{d x d Q^{2}} \cdot \frac{Q^{4} x}{2 \pi \alpha^{2} Y_{+}}=F_{2}\left(x, Q^{2}\right)-\frac{y^{2}}{Y_{+}} \cdot F_{L}\left(x, Q^{2}\right)$

where $\alpha$ is the fine structure constant and $Y_{+}=1+(1-y)^{2}$. The inelasticity $y$ is related to $Q^{2}$, to the centre of mass energy squared of the electron ${ }^{1}$-proton system $s$ and to the Bjorken scaling variable $x$ by $y=Q^{2} / s x$. The expression in equation 1 is often called reduced cross section $\left(\sigma_{r}\right)$. The proton structure function $F_{2}\left(x, Q^{2}\right)$, which provides information on the total quark content of the proton, makes the dominant contribution to the cross section. The longitudinal

\footnotetext{
${ }^{1}$ The term electron in the text is used to denote both electrons and positrons.
}

structure function, $F_{L}\left(x, Q^{2}\right)$, gives a sizable contribution to the cross section only at large values of the inelasticity $y$ since its contribution to the cross section is proportional to $y^{2}$. In lowest order QCD, $F_{L}$ takes the value of zero but due to gluon radiation gets a non-zero value in perturbative QCD (pQCD). Measurements of $F_{L}$ can thus provide constraints on the gluon contribution which is also constrained by the scaling violations of $F_{2}$ as described by the DGLAP QCD evolution equations [1]. An independent measurement of $F_{L}$ at HERA, and its comparison with predictions derived from the gluon distribution extracted from the $Q^{2}$ evolution of $F_{2}$, thus represents a crucial test on the validity of perturbative QCD at low Bjorken $x$. The measurements of $F_{L}$ requires several sets of DIS cross sections at fixed $x$ and $Q^{2}$ but different $y$ which was achieved at HERA in 2007 by variations of the proton beam energy keeping the lepton beam energy fixed.

For $Q^{2} \leq 2 \mathrm{GeV}^{2}$ non-perturbative effects begin to dominate since higher order corrections to the perturbative expansion become large as the strong coupling constant $\alpha_{s}\left(Q^{2}\right)$ increases; this leads to the breakdown of $\mathrm{pQCD}$ calculations. A study of the transition region probes soft hadronic physics, which is currently described by QCD inspired or by purely phenomenological models. To provide a better understanding of soft hadronic processes and to study these models, it is thus important to perform high precision measurements of $e p$ scattering cross sections over the complete transition region. 


\section{COMBINATION OF H1 AND ZEUS DIS $e^{ \pm} p$ SCATTERING CROSS SEC- TION MEASUREMENTS}

DIS cross section measurements obtained from HERA I data previously published by the $\mathrm{H} 1$ and ZEUS collaborations [2-5] have been combined. These data yielded the first accurate DIS cross section measurement from about $115 \mathrm{pb}^{-1}$ of $\mathrm{e}^{ \pm} \mathrm{p}$ scattering data per experiment. The goal of the combined analysis is to provide data of the highest possible accuracy in order to achieve precise determination of the proton parton density functions (PDFs). The analysis is restricted to data with momentum transfers $Q^{2} \geq 1.5 \mathrm{GeV}^{2}$. A significantly reduced overall cross section uncertainty is achieved by taking into account the systematic error correlations in a coherent approach by cross calibrating the various data sets. Since the data precision below $Q^{2} \simeq 200 \mathrm{GeV}^{2}$ is limited by systematic uncertainties, the cross calibration provides a means of substantially reducing the overall uncertainty to below the simple average.

The cross section data are averaged in a model independent way based on reference [6]. In this method for each $\left(x, Q^{2}, y\right)$ value, an averaged value of the cross section at that kinematic point is determined in a $\chi^{2}$ minimization over all measurements allowing the amount of systematic error sources to float.

The combined data for neutral current $\mathrm{e}^{+} \mathrm{p}$ scattering are shown in figure 1. Systematic uncertainties are reduced for the combined data. In the region $Q^{2}<12 \mathrm{GeV}^{2}$ the published $\mathrm{H} 1$ and ZEUS data each have a precision of 2-3\% wheres the combined data set reaches a precision of better than $2 \%$. At larger $Q^{2}$ up to approximately $60 \mathrm{GeV}^{2}$, a precision of $1.5 \%$ is achieved. At the highest $Q^{2}$ the combined data have a significantly reduced total uncertainty of order $10 \%$ which is driven by the statistical accuracy which dominates the measurement error. The complete $\mathrm{e}^{+} \mathrm{p}$ neutral current data shown in figure 1 demonstrate the scaling violations at both high and low $x$. In the figure, the data are shown in comparison to the QCD fit performed by combined data (next section).

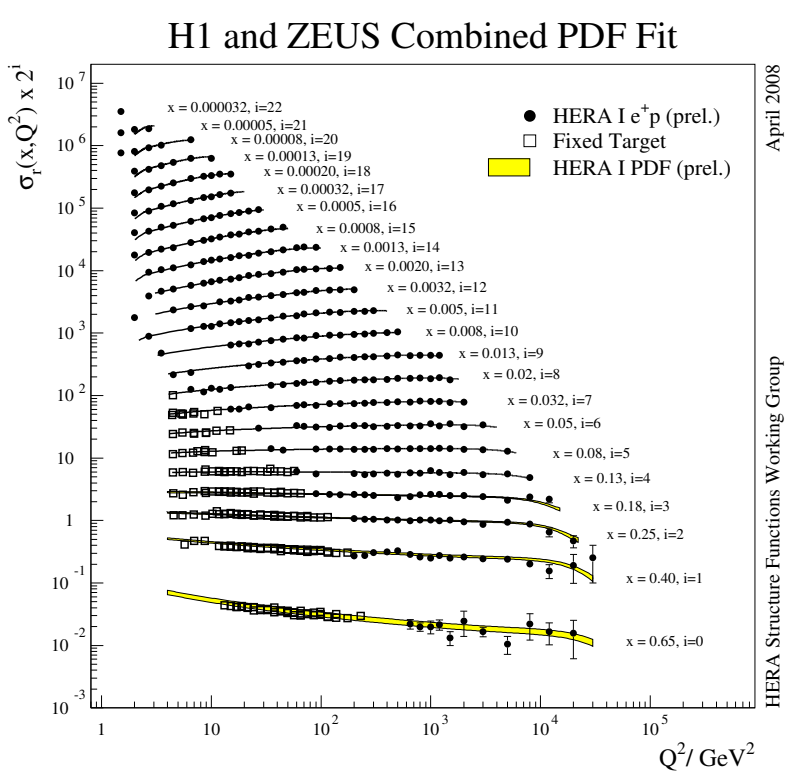

Figure 1. Neutral current DIS $e^{+} p$ reduced cross section measurements from the HERA I data taking period as obtained by combining the published $\mathrm{H} 1$ and ZEUS measurements. The curve is NLO QCD fit performed by combined data.

\section{COMBINED H1 AND ZEUS QCD FIT}

DIS cross sections are the most complete basis for the extraction of parton distributions. The combined results, shown in the previous section, allow a complete set of up and down quark and anti-quark distributions to be determined across the full range of Bjorken $x$ and $Q^{2}$ covered by the measurements and in particular they allow the gluon distribution and, later, the strong coupling constant to be determined with unique accuracy.

Figure 2 shows the $x$ dependence of the $\mathrm{u}$ and $\mathrm{d}$ valence quark as well as sea and gluon distribution functions for $Q^{2}=10 \mathrm{GeV}^{2}$, obtained from the NLO QCD fit performed by combined data. Shadowed regions represent the experimental and model uncertainties. Sea and gluon contributions are scaled down by a factor 0.05 . In comparison with previous fits performed by H1 and ZEUS to their own data $[4,7]$, there is a reduction of the uncertainties achieved, especially in the low $x$ region. 


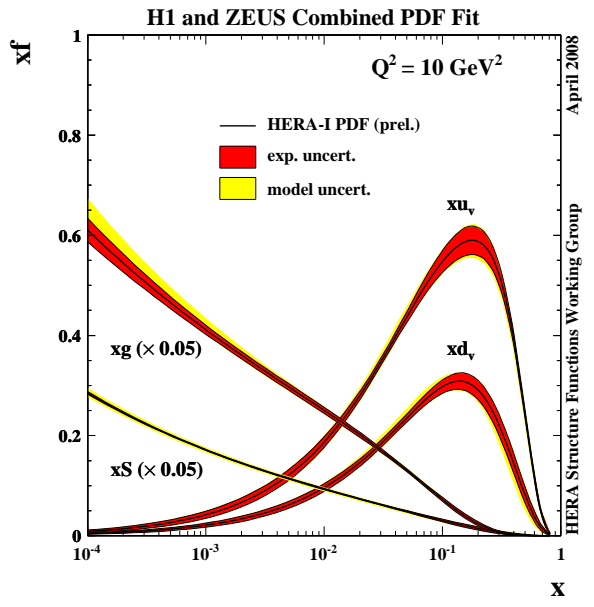

Figure 2. $x$ dependence of the $\mathrm{u}$ and $\mathrm{d}$ valence quark as well as sea and gluon distribution functions for $Q^{2}=10 \mathrm{GeV}^{2}$, obtained from the NLO QCD fit performed by combined HERA I data. Shadowed regions represent the combined experimental and model uncertainties.

\section{NEUTRAL CURRENT CROSS SEC- TION MEASUREMENT AT VERY LOW $Q^{2}$}

In this paper a new measurement of the inclusive $e p$ cross section in the range $Q^{2} \leq 12 \mathrm{GeV}^{2}$ and $5 \cdot 10^{-6} \leq x \leq 0.02$ is presented. The data was collected with the H1 detector in two positron-proton running periods with dedicated settings of the inclusive positron triggers. One data set was collected in 1999 and corresponds to an integrated luminosity of $2.1 \mathrm{pb}^{-1}$. The other was collected in 2000 , with the interaction vertex shifted along the proton beam direction by +70 $\mathrm{cm}$, and corresponds to $505 \mathrm{nb}^{-1}$. This shifting of the interaction region provided detection of the scattered electron at larger polar angles with respect to the incoming proton direction. In this way events with $Q^{2} \leq 2 \mathrm{GeV}^{2}$ are detected within the $\mathrm{H} 1$ detector. These measurements are combined with previously published data [2] taken at a proton beam energy of $820 \mathrm{GeV}$ in the region $Q^{2} \geq 2 \mathrm{GeV}^{2}$ with a luminosity of $1.8 \mathrm{pb}^{-1}$. The combination takes into account

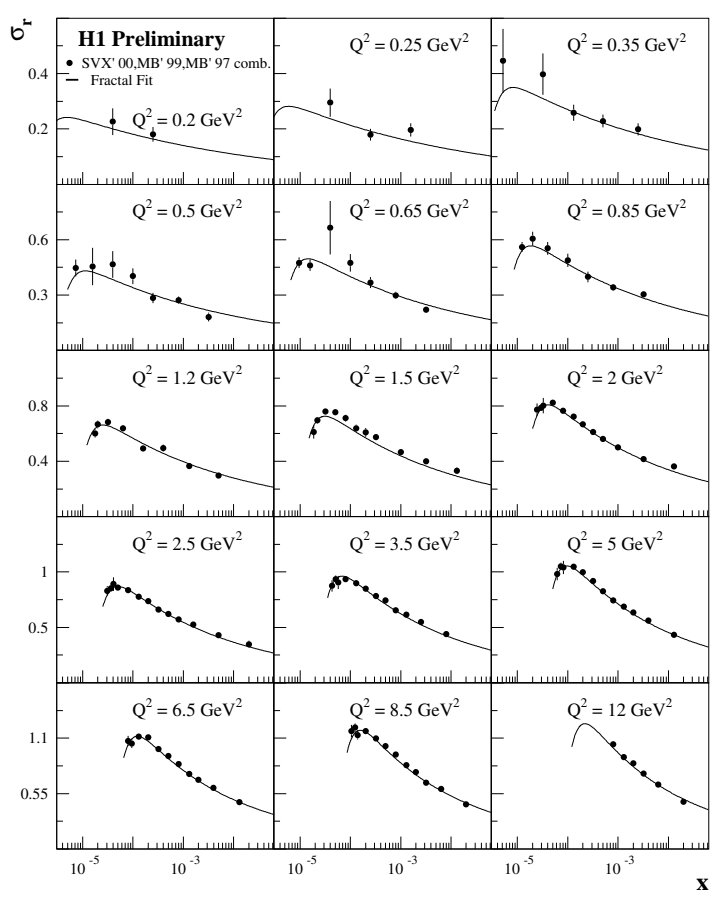

Figure 3. The reduced cross section measured at the $\mathrm{H} 1$ experiment in the region of very low $Q^{2}$ as a function of $x$ compared to the fractal model fit. The errors represent the statistical and systematic errors added in quadrature.

the correlations of the systematic uncertainties.

Figure 3 shows the $x$ dependence of the combined $\mathrm{H} 1 \mathrm{ep}$ reduced cross section for $Q^{2}$ ranging from $0.2 \mathrm{GeV}^{2}$ to $12 \mathrm{GeV}^{2}$. The measurement is compared to the fractal model as introduced in [8]. The total accuracy of the combined datasets is $2-3 \%$ in the bulk region of the measurement. For $Q^{2}>5 \mathrm{GeV}^{2}$ the precision reaches $1.5 \%$. At lowest $x$, corresponding to high $y$, one observes a turn over of $\sigma_{r}$ as is expected from the negative contribution of $F_{L}$ to the cross section, see equation 1.

\section{MEASUREMENT OF $F_{L}\left(x, Q^{2}\right)$}

The sensitivity to the longitudinal structure function $F_{L}$ is largest at high $y$. For a given 
value of $Q^{2}$, measurements at high values of $y$ correspond to low scattered electron energies.

At high $y$ photoproduction often leads to low energy deposits in the calorimeter which fake the scattered electron's signature. Since the photoproduction cross section is much greater than that of DIS, it contributes as the largest source of background. The removal of this background provides the main difficulty for high $y$ measurements and makes a measurement of $F_{L}\left(x, Q^{2}\right)$ a particularly challenging task. To obtain cross section measurements at high $y$ and to test a possibility of measuring $F_{L}\left(x, Q^{2}\right)$, H1 and ZEUS, performed analysis from high statistics data samples collected before 2007, with proton beam energy of $920 \mathrm{GeV}$. As can be seen from references [9] and [10], H1 and ZEUS can perform high $y$ measurements with reasonable accuracy, which is of great importance for the $F_{L}$ measurement.

The first measurement of the longitudinal structure function was performed by H1 collaboration ${ }^{2}$. The longitudinal structure function is obtained from the measurements of the reduced cross section, equation 1 , as the slope of $\sigma_{r}$ versus $y^{2} / Y_{+}$. To get the slope three data sets with different proton beam energies (different $s$ ) provided from HERA in 2007 were used.

The $\mathrm{H} 1$ measurement of $F_{L}\left(x, Q^{2}\right)$ is shown in figure 4. It spans a range in $Q^{2}$ from 12 to $650 \mathrm{GeV}^{2}$. The result is consistent with the prediction obtained with the H1 PDF 2000 fit [4], the last fit from H1 collaboration performed by H1 data, which used only the high energy cross section data of $\mathrm{H} 1$ previously obtained. The measurement is also consistent with previous determinations of $F_{L}$ by $\mathrm{H} 1$ [2], which used NLO QCD to describe and subtract the $F_{2}$ term from the measured reduced cross section at high $y$.

The values of $F_{L}\left(x, Q^{2}\right)$ resulting from averages over $x$ at fixed $Q^{2}$ are presented in figure 5 . The measurement of $F_{L}\left(x, Q^{2}\right)$ is compared with the H1 PDF 2000 fit and with the expectations from global parton distribution fits at higher order perturbation theory by the MSTW group [11] and the CTEQ group [12]. Within the experimental

\footnotetext{
${ }^{2}$ Shortly after this presentation was given first results on $F_{L}$ from ZEUS based on their $460 \mathrm{GeV}$ and $920 \mathrm{GeV}$ data were released and presented at the DIS08 conference [13]
}

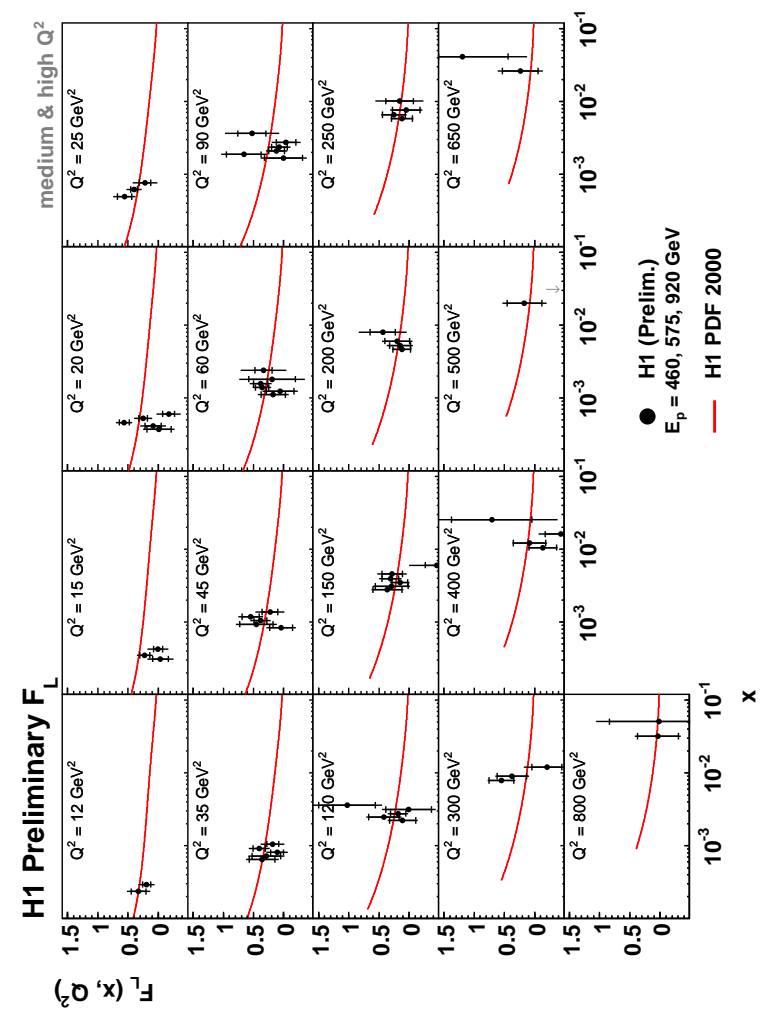

Figure 4. The longitudinal proton structure function. The inner error bars represent the statistical error, the full error bars represent the total errors. The curve represents the NLO QCD prediction derived from the H1 PDF 2000 fit to previous data.

uncertainties the data are consistent with these predictions. The measurement is therefore an important confirmation of the DGLAP evolution framework of perturbative QCD at low Bjorken $x$ at HERA.

The shown $F_{L}\left(x, Q^{2}\right)$ results are obtained from 2007 data with the integrated luminosity of $21.6 \mathrm{pb}^{-1}\left(46.3 \mathrm{pb}^{-1}\right), 12.4 \mathrm{pb}^{-1}\left(12.0 \mathrm{pb}^{-1}\right)$ and $6.2 \mathrm{pb}^{-1}\left(6.2 \mathrm{pb}^{-1}\right)$ for medium (high) $Q^{2}$ measurements for proton beam energy of 920, 460 and $575 \mathrm{GeV}$ respectively.

\section{SUMMARY}

DIS cross section measurements previously published by the $\mathrm{H} 1$ and ZEUS collaborations 


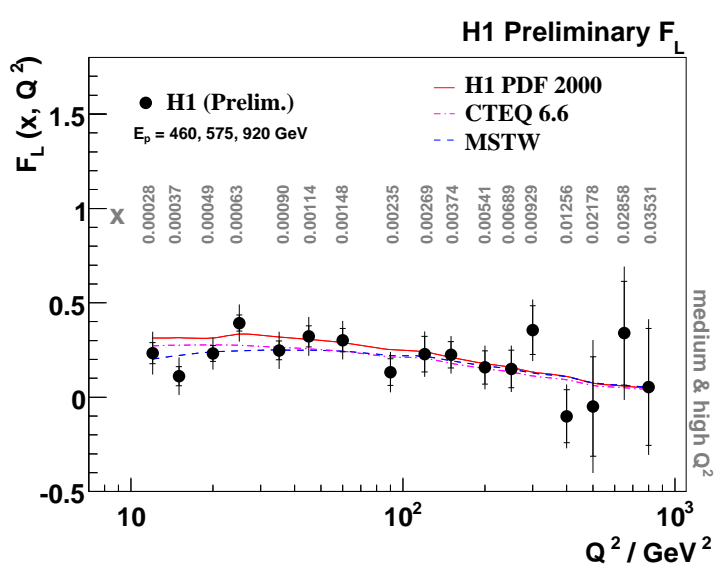

Figure 5. The longitudinal proton structure function $F_{L}$ shown as a function of $Q^{2}$ at the given values of $x$. The inner error bars represent the statistical errors, the full error bars represent the total errors. The solid curve describes the expectation on $F_{L}\left(x, Q^{2}\right)$ from the H1 PDF 2000 fit using NLO QCD. The dashed (dashed-dotted) curve is the expectation of the MSTW (CTEQ) group using NNLO (NLO) QCD.

have been combined. At low and medium $Q^{2}$ a precision better than $2 \%$ is achieved. The combined results provided determination of a complete set of PDFs with unique accuracy. High statistics data from the HERA II running period are being analyzed by the two collaborations and will be included in subsequent combination analysis devoted to precision determination of the parton distributions in the proton.

In the lowest $Q^{2}\left(0.2 \leq Q^{2} \leq 12 \mathrm{GeV}^{2}\right)$ and low Bjorken $x\left(5 \cdot 10^{-6} \leq x \leq 0.02\right)$ regions, preliminary measurements were made using three data sets from $\mathrm{H} 1$ collected during the HERA-I period. The precision of the reduced cross section measurement is $2-3 \%$ in the bulk region, while in the region of $Q^{2}>5 \mathrm{GeV}^{2}$ the precision reaches $1.5 \%$.

The first measurement of the longitudinal structure function in DIS confirm DGLAP QCD predictions for $F_{L}\left(x, Q^{2}\right)$, determined from previous HERA data, which are dominated by a large gluon density at low $x$. At the current level of accuracy, for the covered $Q^{2}$ range between 12 and $800 \mathrm{GeV}^{2}$, a good agreement is thus observed with perturbative QCD.

With the results presented here and further data from HERA II being analyses, the HERA collider experiments are in the process of completing their measurements. For the inclusive cross sections as indicated above the prospects are good to reach a nearly $1 \%$ accuracy level and determine the partonic content of the proton well. This is of importance for QCD and to predict $p p$ scattering results at the LHC.

\section{REFERENCES}

1. V.N. Gribov and L. N. Lipatov, Sov. J. Nucl. Phys. 15 (1972) 438;

V. N. Gribov and L. N. Lipatov, Sov. J. Nucl. Phys. 15 (1972) 675;

Y. L. Dokshitzer, Sov. Phys. JETP 46 (1977) 641 ;

G. Altarelli and G. Parisi, Nucl. Phys. B 126 (1977) 298.

2. C. Adloff et al., Eur. Phys. J. C 21 (2001) 33.

3. C. Chekanov et al., Eur. Phys. J. C 21 (2001) 443.

4. C. Adloff et al., Eur. Phys J. C 30 (2003) 1.

5. C. Chekanov et al., Phys. Rev. D 70 (2004) 052001.

6. A. Glazov, AIP Conf.Proc. 792 (2005) 237.

7. C. Chekanov et al., Eur. Phys. J. C 42 (2005) 1 .

8. T. Lastovicka Eur. Phys. J. C 24 (2002) 529.

9. H1 Collaboration, Munich 2007, Deepinelastic scattering, vol. 1 (2007) 293.

10. ZEUS Collaboration, Munich 2007, Deepinelastic scattering, vol. 1 (2007) 289.

11. A. D. Martin, W. J. Stirling, R. S. Thorne and G. Watt, Phys. Lett. B652 (2007) 292.

12. J. Pumplin, H. L. Lai and W. K. Tung, Phys. Rev. D 75 (2007) 054029;

P. M. Nadolsky et al., hep-ph/0802.0007.

13. ZEUS Collaboration, Presented at DIS08 Workshop, 7-11 April 2008, London, to be published. 